\title{
Melhora dos sintomas do climatério por meio da atividade física: uma revisão sistemática
}

\author{
The improvement of symptoms of climacteric through \\ physical activity: a systematic review
}

TATIANA DE BEM FretTA Universidade Estadual de Santa Catarina, Florianópolis, SC, Brasil.

Nycolle Martins Reis Universidade Estadual de Santa Catarina, Florianópolis, SC, Brasil

Dra. Zenite Machado Universidade Estadual de Santa Catarina, Florianópolis, SC, Brasil

Dra. Adriana Coutinho DE Azevedo Guimarães:

Universidade do Estado de Santa Catarina. Florianópolis, SC, Brasil.

\section{Resumo}

Objetivo: Avaliar a qualidade de vida e a importância da atividade física na melhora dos sintomas do climatério nas mulheres entre 40-65 anos de idade.

Métodos: Trata-se de uma revisão sistemática, cuja estratégia de busca utiliza em cada base de dados a biblioteca eletrônica PubMed/Medline, Lilacs e Scielo "menopause" OR "climacteric" AND "physical activity" AND "quality of life", nos idiomas inglês, português e espanhol entre 2004 e 2016. Foram excluídos os estudos de monografias, teses, dissertações, resumos, revisões sistemáticas e artigos de opinião.

Resultados: Foram encontrados 234 artigos, destes foram selecionados 11 artigos que se encaixaram nos critérios inclusão/exclusão. Os estudos analisados verificaram o efeito da atividade física na melhora dos sintomas do climatério e melhora da qualidade de vida.

Conclusão: A atividade física influencia na melhora dos sintomas do climatério e melhora a estabilidade psicológica e a autoestima, relacionados à qualidade de vida.

Palavras-chave: Climatério. Atividade física E QUalidade De VIDA.

\begin{abstract}
Objective: To evaluate the quality of life and the importance of physical activity in improving symptoms of climacteric in women between 40-65 years of age.

Methods: This is a systematic review, the search strategy used in each database and electronic library PubMed / Medline, Lilacs and Scielo "menopause" OR "climacteric" AND "physical activity" AND "quality of life", in languages English, Portuguese and Spanish between 2004 and 2016 studies were excluded monographs, theses, dissertations, abstracts, systematic reviews and opinion articles.

Results: We found 234 articles, of which 11 were selected articles that fulfilled the criteria inclusion / exclusion. The analyzed studies verified the effect of physical activity in improving climacteric symptoms and improve quality of life.

Conclusion: Physical activity influences the improvement of climacteric symptoms and improve the psychological stability and self-esteem, related to quality of life.
\end{abstract}

Key-words: Climacteric. Physical aCtivity and Quality of Life. 


\section{INTRODUÇÃO}

As mulheres ao longo da vida passam por algumas manifestações marcantes, entre as quais se destaca a menarca e o climatério. Enquanto, a primeira marca o início da fase reprodutiva que costuma acontecer por volta dos 12-13 anos de idade, ${ }^{1}$ o climatério assinala uma mudança da vida reprodutiva para a não reprodutiva, aproximadamente dos 45 aos 65 anos de idade, em razão do declínio gradual da função ovariana. Após um ano de amenorreia, é diagnosticada a menopausa, que é precedida por um período de transição definido como pré-menopausa, onde ocorrem várias transformações físicas, emocionais e sexuais. O período que sucede a última menstruação é denominado pós-menopausa, dessa forma, $o$ período entre a pré-menopausa e a pós-menopausa é denominado climatério. ${ }^{2,3}$

Neste estudo, será enfatizado o climatério, durante esse período crítico na vida das mulheres conhecido por instabilidade hormonal e emocional, ${ }^{1,4}$ devido à diminuição dos hormônios estradiol e progesterona. ${ }^{4,5}$ Segundo Nakano et al., ${ }^{6}$ os sintomas mais corriqueiros são as ondas de calor, afetando $75 \%$ das mulheres nessa fase. Os sintomas vasomotores de calor e suores noturnos são as razões que levam à ausência de sono dessas mulheres. ${ }^{7}$ Devido à deficiência de estrógeno, ocorre um aumento de gordura corporal, principalmente abdominal, elevando o risco de doenças cardiovasculares. ${ }^{8,9} \mathrm{Se}$ gundo Meirelles, ${ }^{9}$ a síndrome metabólica eleva o risco de mortalidade nas mulheres no período da pós-menopausa. Outras queixas comuns nesse período são a irritabilidade, ansiedade, depressão, parestesia, incontinência urinária e as disfunções sexuais, como, alterações do desejo, da excitação e do orgasmo. ${ }^{3,4,5,10,11}$
Devido aos sinais e sintomas característicos desse período, as mulheres parecem ter uma qualidade de vida inferior e baixos níveis de capacidade física. A atividade física pode ser uma aliada na prevenção da sintomatologia, pois promove um efeito protetor sobre o sistema cardiovascular, reduzindo a hipertensão arterial nas mulheres nessa fase da vida. ${ }^{12}$

A atividade física estimula a secreção de endorfinas hipotalâmicas, substâncias essas envolvidas na termorregulação hipotalâmica, que reduzem os sintomas vasomotores e promovem o fortalecimento muscular, atuam também na manutenção da mobilidade articular e na capacidade respiratória, além de diminuir o acúmulo de gordura corporal. ${ }^{10}$ Por fim, a atividade física contribui para melhorar a imagem corporal, elevando a autoestima feminina e, por conseguinte, resultando em uma qualidade de vida positiva. ${ }^{10}$

Sendo assim, este estudo objetivou revisar sistematicamente os estudos transversais, randomizados e experimentais que buscaram avaliar os efeitos da atividade física na melhora dos sintomas do climatério e na qualidade de vida.

\section{MÉTOdo}

O presente estudo caracteriza-se como uma revisão sistemática de trabalhos científicos que estudaram a relação entre o climatério, atividade física e qualidade de vida. Com o intuito de apresentar de forma técnica a redação dos dados, optou-se por seguir as recomendações do PREFERRED REPORTING ITEMS FOR SYSTEMATIC REVIEWS AND META-ANÁLISE - PRISMA. ${ }^{13}$ Foram selecionados por meio de busca eletrônica os artigos das bases de dados PubMed, LILACS (Literatura Latino-Americana e do 
Caribe em Ciências da Saúde) e na biblioteca virtual SciELO (Scientific Electronic Library Online), publicados nos últimos doze anos (2004 a 2016), na área 21 da CAPES com Qualis A1 a B2 e/ou fator de impacto 1.04 a 2.356. A busca dos estudos foi conduzida entre julho a dezembro de 2016.

As palavras-chave utilizadas para buscar os estudos, conforme os descritores em Ciências da Ssaúde (DECs) foram os seguintes: menopausa, climatério, atividade física e qualidade de vida, presentes no título, nos resumos dos artigos e nas palavras-chaves. Mesmo sabendo que não se tratam de sinônimos foram incluídos os termos climatério e menopausa, pois se observou nos estudos uma confusão/mistura nesses termos. Para a combinação dos termos, empregou-se o operador booleano AND e OR. A estratégia de busca utilizada em cada base de dados e biblioteca eletrônica é apresentada a seguir: PubMed/Medline "menopause" OR "climacteric" AND "physical activity" AND "quality of life", Lilacs "menopause" OR "climacteric" AND "physical activity" AND "quality of life", SciELO "menopausa" OR "climatério" AND "atividade física" $A N D$ "qualidade de vida".

Foram considerados como critério de inclusão os artigos em português, inglês e espanhol, que envolvem os descritores já citados, disponíveis em formato digital com delineamento transversal, observacional, ensaio clínico randomizado, quase experimental, estudo de coorte, estudo prospectivo, e/ou epidemiológico que estudaram amostras com idade entre 40 a 65 anos no climatério que tenham utilizado alguma medida, direta e/ou indireta para avaliar a atividade física e a qualidade de vida.

Nesta primeira etapa do estudo, foram identificados os títulos e resumos, além de uma busca secundária na lista bibliográfica dos artigos. Foram excluídos os estudos de monografias, teses, dissertações, resumos, revisões sistemáticas e artigos de opinião. Após a exclusão dos artigos que não cumpriram os critérios de inclusão, com os artigos duplicados foram realizados os fichamentos de todos os artigos selecionados para organização da leitura integral. Todo o processo de busca nas bases de dados eletrônicos, seleções dos estudos, leitura dos artigos, compilação das informações e avaliação metodológica foram efetuados por duas pesquisadoras de forma independente. Diante de discordância, a inclusão do estudo em questão foi resolvida por um terceiro pesquisador.

Utilizou-se a escala proposta por Downs e Black, ${ }^{14}$ composta por 27 itens: comunicação, validade externa, validade interna (viés), validade interna (fatores de confusão) e poder estatístico para a avaliação metodológica dos estudos que realizaram intervenção. Para cada questão, o escore zero será atribuído caso o artigo não atenda aos requisitos da avaliação e o escore será 1 caso ele atenda. Somente a questão 5 atribui a 2 escores, com a seguinte questão "a distribuição dos principais fatores de confusão em cada grupo de indivíduos a serem comparados está claramente descrita? Uma lista dos principais fatores de confusão é apresentada". Com isso, a pontuação máxima a que poderia chegar cada artigo é de 28 pontos.

Para os estudos sem intervenção, optou-se por utilizar uma versão adaptada da mesma escala, já empregada por outros estudos nacionais de revisão sistemática, ${ }^{15,16}$ na qual foram retiradas as questões $4,8,13$ a $15,19,23,24$, porque se referiam aos estudos do tipo ensaio clínico. Foram avaliados, desse modo, 19 itens, que finalizaram em uma pontuação de 20 pontos. 
Os principais resultados extraídos foram o delineamento dos estudos, o número dos sujeitos, os instrumentos empregados para avaliar o climatério, a atividade física e a qualidade de vida, bem como, as covariáveis e os principais achados para expressar os resultados desses estudos. Foram utilizadas medidas de prevalência, média e desvio padrão, $O R$ (odds ratio) e risco relativo.

\section{Resultados}

A Figura 1 apresenta o fluxograma da estratégia utilizada para a seleção dos ar- tigos. Após a busca inicial, foram encontrados 234 artigos com resumo disponível, 3 em espanhol, 11 em português e 220 em inglês, sendo 220 no PubMed, 9 no Lilacs e 5 Scielo.

No entanto, 11 estudos foram selecionados, segundo os critérios preestabelecidos, os demais foram excluídos.

Do total, foram excluídos 42 por serem publicados fora da data definida nos métodos, 10 por não oferecerem artigos completos, 168 por não serem referentes ao tema, por fim, 1 por consistir referências cruzadas.

Figura 1. Fluxograma da seleção dos estudos de acordo com as diretrizes PRISMA

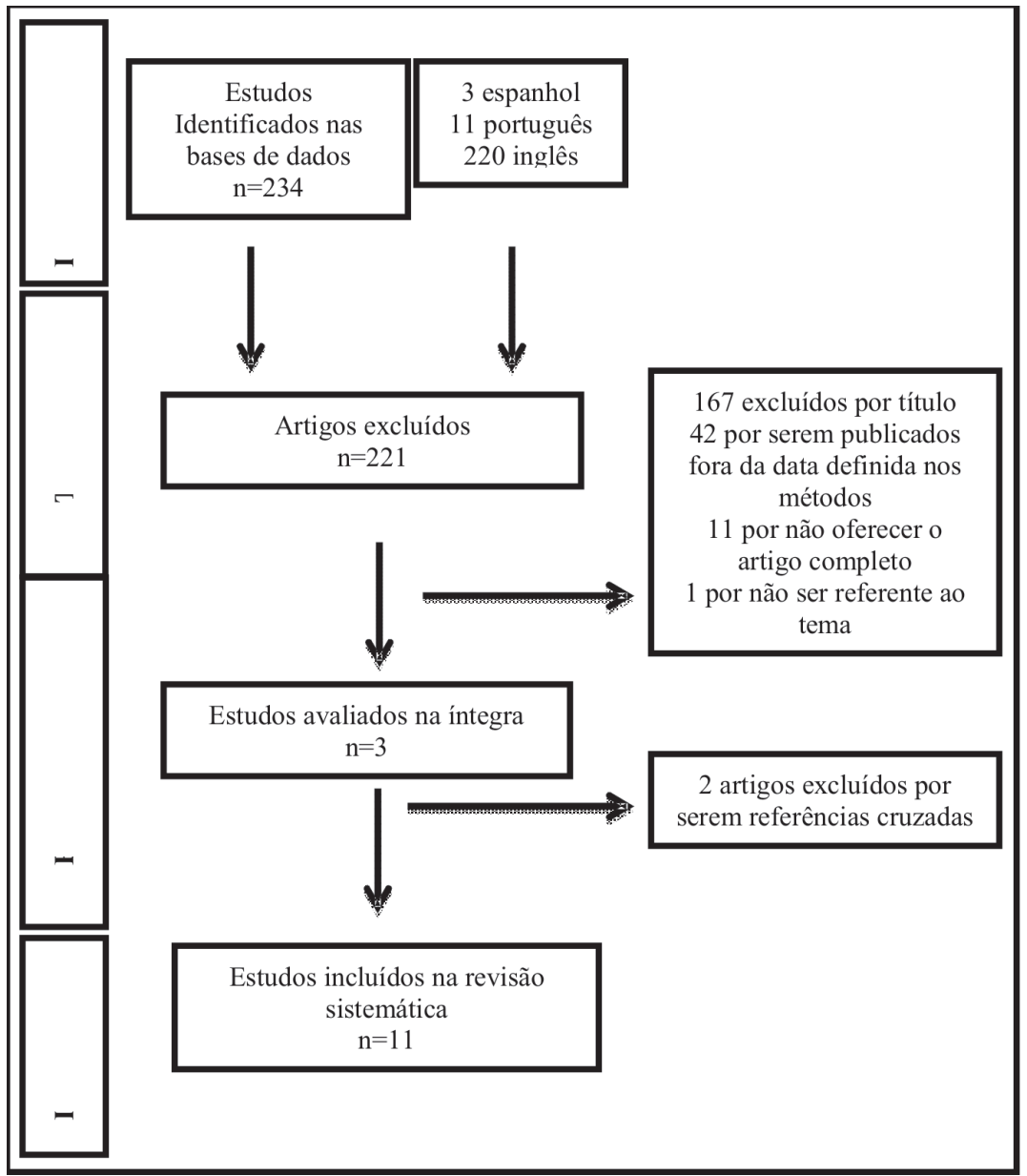


A maioria dos artigos selecionados foram publicados em língua inglesa (10), com predomínio à base de dados PubMed (10) e um artigo publicado em língua portuguesa. Os achados evidenciaram que os anos predominantes de publicação foram $2011 \mathrm{e}$ 2012, ambos com duas publicações, sendo um periódico de Revista Brasileira.

Dos 11 estudos selecionados, todos especificaram os materiais e métodos, estudo transversal $(\mathrm{n}=2)$, descritivo analítico $(\mathrm{n}=1)$, estudo de base populacional $(\mathrm{n}=1)$, estudo controlado ( $\mathrm{n}=1)$, ensaio clínico randomizado e controlado $(n=3)$. A amostra variou de 35 a 2.399 mulheres, totalizando 5.454 mulheres envolvidas, a faixa etária variou entre 40 e 65 anos.

A Tabela 1 foi construída para a integração dos artigos encontrados, identificando a atividade física e a qualidade de vida no climatério, o tipo de estudo, a amostra apresentada, a idade, o instrumento utilizado para a realização do estudo, os resultados correspondentes e o escore.

Tabela 1. Análise dos estudos quanto ao tipo, amostra, idade, instrumentos, atividade física, resultados e escore.

\begin{tabular}{|c|c|c|c|c|}
\hline ESTUDO & $\begin{array}{l}\text { PACIENTES/ } \\
\text { IDADE }\end{array}$ & INSTRUMENTOS/AF & RESULTADOS & ESCORE \\
\hline $\begin{array}{l}\text { Dabrowska et } \\
\text { al. (2016) } \\
\text { Ensaio clínico } \\
\text { randomizado }\end{array}$ & $\begin{array}{l}80 \text { mulheres } \\
\text { rurais. } \mathrm{GC}=40 \\
\text { mulheres e } \\
\mathrm{GI}=40 \text { mulheres }\end{array}$ & $\begin{array}{l}\text { QV SF-36 } \\
\text { GI realizaram AF 3X/ } \\
\text { semana com duração de } \\
60 \text { minutos, os exercícios } \\
\text { eram alongamentos, } \\
\text { caminhada e exercícios } \\
\text { com elástico. }\end{array}$ & $\begin{array}{l}\text { O GI comparado com GC melhorou a QV } \\
\text { significativamente nos domínios vitalidade } \\
\text { ( } \mathrm{p}=0,0046) \text { e na saúde mental ( } \mathrm{p}=0,0052) \text {. } \\
46,25 \% \text { das mulheres tinham o ensino } \\
\text { primário, } 52,50 \% \text { com excesso de peso e } \\
88,75 \% \text { eram casadas. }\end{array}$ & $19^{* *}$ \\
\hline $\begin{array}{l}\text { Mansikkamaki } \\
\text { et al. (2015) } \\
\text { Ensaio clínico } \\
\text { randomizado }\end{array}$ & $\begin{array}{l}159 \text { mulheres, } \\
\text { após } 2 \text { anos } \\
\text { e meio } 102 \\
\text { mulheres e } \\
\text { após } 4 \text { anos } 95 \\
\text { mulheres. } \\
58 \text { anos }\end{array}$ & $\begin{array}{l}\text { QV SF-36 } \\
\text { Acelerômetro para } \\
\text { avaliar a AF. } \\
\text { O GI realizou } \\
\text { treinamento aeróbico } \\
\text { 4X/semana com duração } \\
\text { de } 50 \text { minutos. }\end{array}$ & $\begin{array}{l}\text { O GI relatou melhora na QV e no } \\
\text { funcionamento físico e mental. As mulheres do } \\
\text { GI apresentaram resultados positivos ao longo } \\
\text { dos anos e efeitos na QV depois de } 4 \text { anos. }\end{array}$ & $18^{* \star}$ \\
\hline $\begin{array}{l}\text { Kim et al. } \\
\text { (2014) } \\
\text { Estudo } \\
\text { transversal }\end{array}$ & $\begin{array}{l}631 \text { mulheres } \\
48,5 \pm 2,7 \text { anos }\end{array}$ & $\begin{array}{l}\text { Escala Menopausa } \\
\text { MENQOL, para avaliar } \\
\text { a atividade física IPAQ e } \\
\text { MET min/week. }\end{array}$ & $\begin{array}{l}\text { O IMC foi de } 22,8 \pm 3,1 \mathrm{Kg} .57,8 \%, 29,1 \% \text { e } \\
14,1 \% \text { apresentaram níveis baixo, moderado } \\
\text { e alto de AF respectivamente. A AF era } \\
\text { praticada significativamente nas mulheres } \\
\text { mais novas comparadas com as mulheres de } \\
\text { baixo índice de AF ( } \mathrm{p}=0,011 \text { ). O grupo ativo } \\
\text { teve significativamente menores níveis de } \\
\text { insulina em jejum (HOMA-IR e TG) e níveis } \\
\text { elevados HDL-C comparado com o grupo de } \\
\text { baixa AF. Na análise do MENQOL, as mulheres } \\
\text { que praticam AF moderadamente relataram } \\
\text { significativamente menor escore psicossocial } \\
\text { (p=0,012) e sintomas físicos ( } \mathrm{p}=0,002) \\
\text { comparado com as mulheres de baixa AF. } \\
\text { Não foram observados associações entre a } \\
\text { AF, sintomas vasomotores e sexuais nos três } \\
\text { grupos. }\end{array}$ & $14^{*}$ \\
\hline
\end{tabular}




\begin{tabular}{|c|c|c|c|c|}
\hline ESTUDO & $\begin{array}{l}\text { PACIENTES/ } \\
\text { IDADE }\end{array}$ & INSTRUMENTOS/AF & RESULTADOS & ESCORE \\
\hline $\begin{array}{l}\text { Javadivala et al. } \\
\text { (2013) Estudo } \\
\text { descritivo- } \\
\text { analítico }\end{array}$ & $\begin{array}{l}273 \text { mulheres } \\
49,8 \pm 5,3 \text { anos }\end{array}$ & $\begin{array}{l}\text { Escala Menopause } \\
\text { Rating, atividade física } \\
\text { (AF) pelo IPAQ versão } \\
\text { curta e a qualidade de } \\
\text { vida (QV) WHOQOL. }\end{array}$ & $\begin{array}{l}\text { As mulheres com sinais moderados de } \\
\text { esgotamento físico-mental fizeram mais AF } \\
\text { comparado com as mulheres que tiveram } \\
\text { exaustão severa }(\mathrm{P}=0,006) \text {. No domínio } \\
\text { psicológico, teve um aumento } 0,436 \text { hora de } \\
\text { AF }(\mathrm{P}=0,006) \text {. As mulheres não tinham sinais } \\
\text { de problemas sexuais }(\mathrm{P}<0,048) \text { e realizaram } \\
\text { menos AF comparado com aquelas que tiveram } \\
\text { história grave de problemas sexuais }(\mathrm{P}<0,006) \text {. } \\
\mathrm{E} \text { as com secura vaginal relataram } 3,011 \text { hora a } \\
\text { menos de AF }(\mathrm{P}=0,030) \text { em comparação com } \\
\text { mulheres que tiveram secura vaginal muito } \\
\text { grave. }\end{array}$ & $12^{*}$ \\
\hline
\end{tabular}

\begin{tabular}{lll}
\hline Luoto et al. & GI=74 mulheres & Questionário Saúde \\
(2012) & GC=77 & Feminina (WHQ) e a \\
mulheres & QV SF-36. / Treinamento \\
& $54,5 \pm 3,8$ anos & aeróbico não \\
GI & supervisionado por 50 \\
$54,2 \pm 3,7$ anos & minutos 4X por semana, \\
GC & durante 6 meses.
\end{tabular}

Após 6 meses, as mulheres do grupo de

intervenção tiveram melhor resultados SF-36

comparado com grupo controle em vitalidade

$(\mathrm{P}=0,02)$, saúde mental $(\mathrm{P}=0,02)$, capacidade

física $(\mathrm{P}=0,049)$, limitação do papel físico

$(\mathrm{P}=0,017)$, saúde geral $(\mathrm{P}=0,07)$ e vitalidade

$(\mathrm{P}=0,06)$. WHQ após 6 meses, depressão

$(\mathrm{P}=0,03)$, ansiedade $(\mathrm{P}=0,06)$ e sintomas menstruais $(\mathrm{P}=0,01)$.

\begin{tabular}{ll}
\hline Moilanen & 1.165 mulheres \\
et al. (2012) & $47 \pm 5,2$ anos no \\
Estudo de base & ano de 2000 \\
populacional & $56 \pm 5.2$ anos no \\
nacional & ano de 2008 \\
representativa &
\end{tabular}

representativa

Questionário Health
Study 2000, em 2008
a análise da QV escala
modificada Andrew e
Withley. AF foi medida
pelo MET. Outras
variáveis incluídas
nas análises: idade,
educação, mudança de
AF, mudança de peso e
uso de terapia hormonal
(TRH).
O estado da menopausa não era
significativamente correlacionado com a mudança de QV. QV: as mulheres com alto nível de educação apresentaram menores sintomas da menopausa em relação às menos escolarizadas. As mulheres que aumentaram ou permaneceram estáveis na AF tiveram maiores chances de melhorarem a QV do que as mulheres que diminuíram a sua AF. As mulheres que mantiveram seu peso estável durante os oito anos também melhoraram sua QV em comparação com as mulheres que ganharam peso. As mulheres que nunca usaram TRH tiveram mais chances de melhorar a QV. A melhora da QV global está correlacionada com a sua AF.

\begin{tabular}{ll}
\hline Guimarães \& & 104 mulheres \\
Baptista (2011) & $50 \pm 4,1$ anos \\
Quase- & \\
experimental &
\end{tabular}
Variação média QV e sintomas da menopausa com prática habitual de AF (menos 30min/ dia, entre 30 e $60 \mathrm{~min} /$ dia e $60 \mathrm{~min} /$ dia ou mais) Domínio físico $\mathrm{P}=0,062$, domínio psicológico $\mathrm{P}=0,001$ domínio social $\mathrm{P}=0,035$, houve uma diferença significativa $\mathrm{P}<0,05$ entre o grupo que manteve ou aumentou AF para mais 60 min/dia. Variação média da QV e prática AF, as mulheres tiveram uma diminuição nos sintomas no domínio físico $\mathrm{P}=0,623$, domínio psicológico $\mathrm{P}=0,001$ e domínio social $\mathrm{P}=0,073$. Entre as mulheres sem sintomas decrescente, domínio físico $\mathrm{P}=0,545$, domínio psicológico $\mathrm{P}=0,761$ e domínio social $\mathrm{P}=0,584$, houve uma diferença significativa $\mathrm{P}<0,05$ entre o grupo que manteve ou aumentou AF para mais 60 $\mathrm{min} /$ dia.




\begin{tabular}{|c|c|c|c|c|}
\hline ESTUDO & $\begin{array}{l}\text { PACIENTES/ } \\
\text { IDADE }\end{array}$ & INSTRUMENTOS/AF & RESULTADOS & ESCORE \\
\hline $\begin{array}{l}\text { Gonçalves } \\
\text { et al. (2011) } \\
\text { Estudo de base } \\
\text { populacional } \\
\text { e de corte } \\
\text { transversal }\end{array}$ & $\begin{array}{l}370 \text { mulheres } \\
49,8 \pm 8,1 \text { anos }\end{array}$ & $\begin{array}{l}\text { Whoqo - Bref para } \\
\text { avaliar QV. Menopause } \\
\text { Rating Scale (MRS), para } \\
\text { avaliar os sintomas do } \\
\text { climatério. Ipaq versão } \\
\text { curta, avaliar o nível de } \\
\text { AF. }\end{array}$ & $\begin{array}{l}\text { Os escores para a QV foram diferentes, entre } \\
\text { os grupos sedentários, moderadamente ativas } \\
\text { e muito ativas }(\mathrm{p}<0,01) \text {. As mulheres ativas } \\
\text { tiveram uma melhor QV }(\mathrm{P}<0,01) \text {. Em relação } \\
\text { à AF e os sintomas do climatério, foram } \\
\text { observadas diferenças significativas para todos } \\
\text { os domínios: psicológico }(\mathrm{p}<0,01) \text {, somático- } \\
\text { vegetativo }(\mathrm{p}<0,01) \text { e urogenital }(\mathrm{p}<0,01) \text {. }\end{array}$ & $14^{*}$ \\
\hline $\begin{array}{l}\text { Elavsky (2009) } \\
\text { Estudo } \\
\text { randomizado e } \\
\text { controlado }\end{array}$ & $\begin{array}{l}164 \text { mulheres } \\
42-58 \text { anos }\end{array}$ & $\begin{array}{l}\text { Informações gerais, } \\
\text { padrões de sangramento } \\
\text { Stages of Reproductive } \\
\text { Aging Workshop } \\
\text { (STRAW), Sintomas } \\
\text { da menopausa Greene } \\
\text { Climacteric Scale (GCS), } \\
\text { AF e índice de massa } \\
\text { corporal Aerobics Center } \\
\text { Longitudinal Study } \\
\text { Physical Activity Survey } \\
\text { (ACLS), Afeto positivo } \\
\text { e negativo Affectometer } \\
\text { 2, Autoestima Physical } \\
\text { Self-Worth (PSW) e } \\
\text { QV Quality of life Scale } \\
\text { (UQOL). GI de } 4 \text { meses } \\
\text { de exercícios de yoga } \\
\text { e caminhadas e GC } \\
\text { monitoradas por } 2 \text { anos. }\end{array}$ & $\begin{array}{l}\text { O aumento da AF diminui os sintomas da } \\
\text { menopausa, isso foi relacionado ao aumento } \\
\text { da autoestima e melhora na QV. Os efeitos da } \\
\text { AF sobre a QV têm benefícios a longo prazo } \\
\text { para as mulheres submetidas a transição da } \\
\text { menopausa. }\end{array}$ & $18^{\star *}$ \\
\hline $\begin{array}{l}\text { Daley et al. } \\
(2007)\end{array}$ & $\begin{array}{l}2.399 \text { mulheres } \\
50,5 \pm 2,8 \text { anos }\end{array}$ & $\begin{array}{l}\text { QV questionário } \\
\text { de saúde da mulher } \\
\text { (WHQ). Realizavam } \\
\text { exercícios } 3 X / \text { semana } \\
\text { ou mais duração de } 20 \\
\text { minutos ou mais. }\end{array}$ & $\begin{array}{l}\text { Em relação a AF e a QV, as mulheres ativas } \\
\text { relataram menor escore de depressão } \mathrm{P}<0,001 \text {, } \\
\text { ansiedade } \mathrm{P}<0,01 \text {, sintomas somáticos } \mathrm{P}<0,01 \text {. } \\
\text { As mulheres obesas relataram sintomas } \\
\text { vasomotores mais elevados } \mathrm{P}<0,01 \text { e também } \\
\text { relataram sintomas somáticos } \mathrm{P}<0,01 \text {. }\end{array}$ & $15^{*}$ \\
\hline Ueda (2004) & $\begin{array}{l}35 \text { mulheres } \\
48.4 \pm 9.8 \text { anos }\end{array}$ & $\begin{array}{l}\text { Os sintomas do } \\
\text { climatério foram } \\
\text { avaliados utilizando } \\
\text { a versão japonesa do } \\
\text { Îndice de Kupperman, } \\
\text { QV foi avaliada por meio } \\
\text { do questionário QV } \\
\text { climatério e desenvolvido } \\
\text { por Sugiyama. A } \\
\text { AF, foi avaliada pelo } \\
\text { teste de diagnóstico } \\
\text { comportamento. GI } \\
\text { realizaram AF 3X por } \\
\text { semana e o GC não } \\
\text { praticaram AF. }\end{array}$ & $\begin{array}{l}\text { O programa obteve efeitos significativos } \\
\text { no índice Kupperman, principalmente nos } \\
\text { sintomas psicossomáticos, especialmente, } \\
\text { parestesia e nervosismo, assim como houve } \\
\text { uma melhora nas palpitações. A AF teve um } \\
\text { efeito positivo nos sintomas do climatério. Esse } \\
\text { programa não propiciou resultado na QV. }\end{array}$ & $13^{* *}$ \\
\hline
\end{tabular}

Fonte: Construída pelo próprio autor. AF atividade física. Escore referente à Escala Downs \& Black12. *Pontuação dos artigos sem intervenção máximo: 20 pontos. ${ }^{\star *}$ Pontuação dos artigos com intervenção máximo: 28 pontos. 
Com relação à atividade física realizada, tão-somente, quatro especificaram o tipo de exercício, apenas seis especificaram a frequência e cinco a duração dos exercícios. Verificou-se, que os estudos foram realizados, em sua maioria, no Brasil e na Finlândia, seguido pelo Irã, Estados Unidos, Inglaterra, Japão, Coreia do Sul e Polônia.

No estudo quase experimental ${ }^{20}$ e no ensaio clínico randomizado, ${ }^{18,22}$ foi observado melhora significativa nos sintomas do climatério após o período de intervenção. Isso ocorreu quando se comparou o mesmo grupo no início e no final do programa, bem como, a comparação entre os grupos intervenção e controle. Todos os estudos relataram que quanto mais elevado o nível de atividade física tanto menor os sintomas do climatério. Destaca-se também a influência positiva da atividade física na qualidade de vida das mulheres no climatério nos estudos de coorte e prospectivo. ${ }^{17-24}$

Com relação à avaliação metodológica dos artigos desta revisão, verificou-se entre os estudos que realizaram intervenção, ocorreu uma variação de escore entre 13 a 21 pontos com uma média de 57,75 pontos. Quanto aos estudos que não tiveram intervenção, observou-se uma variação de escore entre 12 a 15 pontos com média de 90,14 pontos.

Os instrumentos utilizados para avaliar o climatério foram: a escala menopause rating scale $(\mathrm{n}=2)$, questionário saúde feminina WHQ $(n=2)$, menopausa de KUPPERMAN $(n=1)$, escala LIKART $(n=1)$, greene climacteric Scale $(\mathrm{n}=1)$, questionário health $(\mathrm{n}=1)$ e stages of reproductive aging workshop STRAW $(\mathrm{n}=1)$. A autoestima physical selfWorth $(\mathrm{n}=1)$ e affectometer $(\mathrm{n}=1)$. Para avaliar a qualidade de vida o WHOQOL $(n=3)$,
SF-36 $(\mathrm{n}=3)$, quality of life scale UQOL $(n=1)$ e análise de qualidade de vida global QOL $(n=1)$ e, por fim, a atividade física por meio do IPAQ versão curta $(\mathrm{n}=4)$, aerobics center longitudinal study physical activity survey ACLS $(\mathrm{n}=1)$ e Tokunaga $(\mathrm{n}=1)$, menopausa MENQOL $(\mathrm{n}=1)$, MET min/week $(n=2)$ e acelerômetro $(n=1)$.

\section{DIscussão}

A partir da literatura analisada neste artigo de revisão, os principais resultados dos estudos destacaram que as mulheres que praticaram atividade física tiveram menor esgotamento físico, sentiram menos ondas de calor e menor tendência à depressão, além de melhorarem o físico e o sistema cardiorrespiratório. Inclusive, aumentaram a massa muscular em relação àquelas sem atividade física, e atingiram um maior escore na qualidade de vida. Mulheres com alto grau de escolaridade e maior renda vivenciaram menor sintomatologia em comparação com as de baixa renda e menor grau de escolaridade. ${ }^{17-27}$

Nos onze estudos que foram parte integrante desta revisão sistemática, observou-se que a atividade física contribuiu para melhorar as condições de saúde das mulheres no climatério. Portanto, as que praticaram atividade física regular ou aumentaram a intensidade do exercício tiveram menos sinais e sintomas em comparação com as que não praticaram atividade física, recomenda-se uma intervenção terapêutica para as mulheres sedentárias. ${ }^{17-24}$

Os estudos investigados evidenciaram que são inúmeros os benefícios que a atividade física promove durante o climatério, 
como a melhora da flexibilidade, maior mobilidade articular, aumento da força muscular, coordenação motora e controle da massa muscular. ${ }^{20,21,22,23,25}$

A intensidade dos sintomas do climatério variou de leve a grave em $96 \%$ das mulheres. As mulheres que realizaram pouca atividade física apresentaram mais sinais de esgotamento físico-mental em comparação com as mais ativas, constatou-se que a atividade física de forma regular gera um impacto positivo na qualidade de vida dessas mulheres. ${ }^{17,18,19,24,25}$ Luoto et al., ${ }^{21}$ encontraram uma associação positiva com o treinamento aeróbico ao verificar que as mulheres que fizeram esse treinamento tiveram melhoras significativas na qualidade de vida, restabelecendo os sintomas de humor e amenizando os sintomas da depressão. As mulheres que realizaram atividade física não tiveram aumento significativo da massa corporal, enquanto que as que não realizaram tiveram um ganho de massa corporal igual ou superior a $5 \mathrm{~kg}$. $\mathrm{O}$ ganho de massa corporal foi acompanhado pelo aumento significativo nos sintomas somáticos, como, dor nas articulações, fadiga, dor abdominal, vertigem e alterações na pele. ${ }^{22,23,26}$

Ressalta-se que as mulheres que realizaram mais atividade física foram as com grau de escolaridade mais elevado e situação econômica mais favorecida. Por terem mais acesso às informações e assistência médica, sentiram menos os sintomas do climatério, além de uma melhora na qualidade de vida em comparação com as de grau de escolaridade mais baixo e situação econômica menos favorecida. ${ }^{22,24,25}$ Nos estudos analisados, a queixa mais comum são os sintomas vasomotores. ${ }^{22,24,26} \mathrm{~A}$ atividade física regular promove um aumento da produção de $\beta$-endorfina hipotalâmica, estabilizando a termorregulação hipotalâmica, sendo assim, aliviando as ondas de calor. ${ }^{22,25,27}$ Esse sintoma interfere no cotidiano das mulheres, no trabalho e na qualidade do sono, ele é o causador de maior desconforto nos níveis de estresse e humor, provocando uma baixa na qualidade de vida. ${ }^{20-26}$ Alguns estudos relataram que as mulheres obesas tiveram mais sintomas vasomotores em comparação com as mulheres de peso normal. ${ }^{22,23,26}$

A atividade física reduz o acúmulo de gordura corporal, melhora o perfil lipídico, a autoestima e a imagem corporal, por conseguinte, melhora o conceito delas sobre si mesmas. ${ }^{22-26}$ Segundo Elavsky, ${ }^{25}$ as mulheres que intensificaram a atividade física constataram aumento da autoestima e afeto positivo. A associação de todos os fatores proporcionou um impacto positivo na qualidade de vida. Os sintomas urogenitais, como a incontinência urinária, secura vaginal, diminuição da libido, diminuição do desejo e insatisfação sexual estão associados à baixa qualidade de vida, de forma que as mulheres que praticaram atividade física, mesmo de baixa intensidade, mencionaram esses sintomas menos severos. ${ }^{23-26}$ Javadivala $^{20}$ obteve um resultado diferente dos estudos anteriores ao constatar que as mulheres com problemas sexuais e secura vaginal realizaram mais atividade física, contrapondo com as mulheres sem esses sintomas.

No estudo realizado por Guimarães e Baptista $^{23}$ com um grupo de mulheres no climatério que praticavam menos de $30 \mathrm{mi}-$ nutos diários de atividade física, e outro, de mulheres que praticavam mais de 60 minutos diários de atividade física, verificou-se que as 
mulheres que realizaram mais atividade física diminuíram os sintomas do climatério, similarmente diminuíram a massa corporal e melhoraram os domínios psicológicos, diante disso, elevaram o escore da qualidade de vida. O climatério está relacionado ao aumento do risco de muitas doenças crônicas incluindo as doenças cardiovasculares. A atividade física regular reduz a frequência cardíaca de repouso, estabilizando a pressão arterial e contribuindo para a menor incidência de doenças cardiovasculares. ${ }^{20}$

O programa realizado por $\mathrm{Ueda}^{27}$ consistia não somente de atividade física, mas de palestras sobre os sintomas e tratamentos do climatério. Essas palestras também abordaram melhoria no estilo de vida, além de aprimorar os conhecimentos adquiridos sobre os sintomas do climatério, e os efeitos benéficos da atividade física nessa fase da vida, de forma geral, incentivou as mulheres a continuarem seus exercícios físicos. No estudo de Luoto, ${ }^{21}$ no qual foi desenvolvido um programa de atividade física de 50 minutos, quatro vezes por semana, durante seis meses, verificou-se que as mulheres do grupo de intervenção tiveram melhor vitalidade e saúde mental, inclusive, melhora do humor, redução da ansiedade, melhora da capacidade física e aumento da massa magra em comparação ao grupo controle.

Entretanto, destaca-se que os 11 estudos realizados usaram medidas frágeis de AF, tornando-se necessárias ações que busquem utilizar estudos controlados randomizados que evitem viés de informações e forneçam evidências mais robustas a respeito de sua influência sobre os sinais e sintomas do climatério.

\section{Considerações Finais}

Pode-se concluir a relevância da $\mathrm{AF}$ para reduzir os sinais e sintomas do climatério, sendo importante incentivar um estilo de vida saudável entre as mulheres. Por fim, sugere-se a realização de outras pesquisas que abordem a temática desenvolvida nesses estudos, a fim de divulgar e conscientizar a população feminina a respeito do climatério e sua relação com a qualidade de vida e os cuidados com a saúde a serem tomados.

\section{REFERÊNCIAS}

1. VITALLE MSS, TOMIOKA CY, JULIANO Y., AMANCIO OMS. Índice de massa corporal, desenvolvimento puberal e sua relação com a menarca. Rev assoc med Bras 2003; 49(4): 429-33.

2. LORENZI DRS, BARACAT EC, SACILOTO B., PADILHA IJ. Fatores associados à qualidade de vida após menopausa. Rev Assoc Med Bras 2006; 52(5): 312-7.

3. LORENZI DRS, SACILOTO B., ÁRTICO GR, FONTANA SKR. Qualidade de vida e fatores associados em mulheres climatéricas residentes na região sul do Brasil. Acta Med Port 2009; 22(1): 51-58.

4. GALVÃO LLLF, FARIAS MCS, AZEVEDO PRM, VILAR MJP, AZEVEDO GD. Prevalência de transtornos mentais comuns e avaliação da qualidade de vida no climatério. Rev Assoc Bras 2007; 53(5): 414-20.

5. GREEN J., DENHAM A., INGRAM J., HAWKEY S., GREENWOOD R. Treatment of menopausal symptoms by qualified herbal practitioners: a prospective, randomized controlled trial. Family Practice 2007; 24: 468-474. 
6. NAKANO K., PINNOW E., FLAWS J., SORKIN JD, GALLICCHIO L. Journal of Women's Health 2012; 21(4): 433-439.

7. LAMBIASE M., THURTON R. Physical activity and sleep among midlife women with vasomotor symptoms. Menopause 2013; 20(9): 946-952.

8. WILLIAMS LT, HOLLIS JL, COLLINS CE, Morgan PJ. The 40-Something randomized controlled trial to prevent weight gain in mid-age women. BMC Public Health 2013; 13(1007): 2-12.

9. MEIRELLES RMR. Menopausa e síndrome metabólica. Rev Bras Endocrinol Metab 2014; 58(2): 91-96.

10. LORENZI DRS, DANELON C., SACILOTO B., PADILHA IJ. Fatores indicadores da sintomatologia climatérica. Rev Bras Ginecol Obstet 2005; 27(1): 12-19.

11. PEDROA AO, NETO AMP, PAIVA LHSC, Osisb MJD, Hardya EE. Síndrome do climatério: inquérito populacional domiciliar em Campinas, SP. Rev. Saúde Púb 2003; 37(6): 735-42.

12. ZANESCO A., ZAROS PR. Exercício físico e menopausa. Rev Bras Ginecol Obstet 2009; 31(5): 254-61.

13. URRÚTIA G., BONFILL X. Declaración PRISMA: uma propuesta para mejorar la publicación derevisiones sistemáticas y metaanálisis. Med Clin 2010; 135(11): 507-11.

14. DOWNS SH, BLACK N. The feasibility of creating a checklist for the assessment of the methodological quality both of randomized and nonrandomized studies of health care interventions. J Epidem Comm Health 1998; 52(52): 377-84.

15. ARAUJO DMR, VILARIM MM, SABROZA AR, NARDI AE. Depressão no período gestacional e baixo peso ao nascer: uma revisão sistemática da literatura. Cad Saúde Pública 2010; 26(2): 219-227.

16. ROSSI CE, VASCONCELOS FAG. Peso ao nascer e obesidade em crianças e adolescentes: uma revisão sistemática. Rev Bras Epidemiol 2010; 13(2): 246-258.

17. DABROWSKA J., DABROWSKA-GALAS M., RUTKWSKA M., MICHALSKI BA. Twelve-week exercise training and the quality of life in menopausal women - clinical trial. Menopause 2016; 15(1): 20-25.

18. MANSIKKAMAKI K., RAITANEN J., NYGARD CH, TOMÁS E., RUTANEN R, LUOTO R. Long-term effect of physical activity on health-related quality of life among menopausal women: a 4-year follow-up study to a randomized controlled trial. BMJ OPEN 2015; 5: 01-08.

19. KIM MJ, CHO J., AHN Y., YIM G., PARK HY. Association between physical activity and menopausal symptoms in perimenopausal women. Women's Health 2014; 14 (122): 02-08.

20. JAVADIVALA Z., KOUSHA A., ALLAHVERDIPOUR H., JAFARABADI MA, TALLEBIAN H. Modeling the Relationship between Physical Activity and Quality of Life in Menopausal-aged Women: A Cross-Sectional Study. JRHS 2013; 13(2): 168-175.

21. LUOTO R., MOILANEN J., HEINONEN R., MIKKOLA T., RAITANEN J., TOMAS E., et al. Effect of aerobic training on hot flushes and quality of life a randomized controlled trial. Annals of Medicine 2012; 44: 616-626.

22. MOILANEN JM, ALTO AM, RAITANEN J., HEMMINKI E., ARO AR, LUOTO R. Physical activity and change in quality of life during menopause an 8 year follow-up study. Health and Quality of Life Outcomes 2012; 10: 8.

23. GUIMARÃES ACA, BAPTISTA F. Influence of habitual physical activity on the symptoms of climacterium/menopause and the quality of life of middle-aged women. Internat Journ of Women's Health $2011 ; 319-28$.

24. GONÇALVES AKS, CANÁRIO ACG, CABRAL PUL, SILVA RAH, SPYRIDES MHC, GIRALDO PC, et al. Impacto a atividade física na qualidade de vida de mulheres de meia idade: estudo de base populacional. Rev Bras Ginecol Obstetr 2011; 33(12): 408-13. 
25. ELAVSKY S. Physical Activity, Menopause, and Quality of Life: The Role of Affect and SelfWorth across Time. Menopause 2009; 16(2): 265-271.

26. DALEY A., MACARTHUR C., LAMPARD HS, MCMANUS R., WILSON S., MUTRIE N. Exercise participation, body mass index, and health-related quality of life in women of menopausal age. British Journal of General Practice 2007; 130-135.

27. UEDA M. A 12-week Structured Education and Exercise Program Improved Climacteric Symptoms in Middle-aged Women. Journ of Physiol anthropol Appl Human Sci 2004; 23: 143-148.

\section{DADOS DA AUTORA}

\section{TAtiana de Bem Fretta}

Mestranda no programa de Pós-graduação em Ciências do Movimento Humano, Centro de Ciências da Saúde e do Esporte do Universidade Estadual de Santa Catarina, Florianópolis, SC, Brasil. Endereço: Trav. Ademir Guimarães, 176 ap 102A, Bairro: João Paulo. Florianópolis, SC- Brasil. CEP:88030420. E-mail: tatibem@hotmail.com (autora para correspondência).

\section{Nycolle Martins Reis}

Mestre no programa de Pós-graduação em Ciências do Movimento Humano, Centro de Ciências da Saúde e do Esporte do Universidade Estadual de Santa Catarina, Florianópolis, SC, Brasil.

\section{Dra. Zenite Machado}

Universidade Estadual de Santa Catarina, Florianópolis, SC, Brasil.

\section{Dra. Adriana Coutinho de Azevedo Guimarães}

Departamento de Educação Física e Programa de Pós-graduação em Ciências do Movimento Humano, Centro de Ciências da Saúde e do Esporte. Universidade do Estado de Santa Catarina. Florianópolis, SC, Brasil. Professora Adjunto da Universidade do Estado de Santa Catarina, Brasil.

Submetido em: 19-04-2017

Aceito em: 21-7-2017 